\section{What is the contribution of physical activity on outcome in patients with type 2 diabetes mellitus?}

\author{
Qual a contribuição da atividade física sobre o desfecho \\ em pacientes com diabetes melito tipo 2?
}

Mustafa Dinc', Sevket Balta'2, Mustafa Cakar', Muharrem Akhan³, Hakan Sarlak ${ }^{3}$, Murat Unlu ${ }^{4}$

$\mathrm{W}$ e read the article "Associations among physical activity, comorbidities, depressive symptoms and health-related quality of life in type 2 diabetes" written by Thiago Medeiros da Costa Daniele and cols. with a great interest (1). They concluded that functional capacity is independently related to physical activity in diabetic patients, and that increasing patient independence and treating depressive symptoms can promote physical activity. We would like to thank the authors for their contribution with a successfully designed and documented study. We believe that these findings will enlighten further studies about physical activity and its role in type 2 diabetes mellitus (T2DM).

This study showed that a significant proportion of diabetic patients had a sedentary lifestyle. Interestingly, diabetic patients were more active than the age-matched control population, and authors considered this finding a result of education. This result is very important and show us precious data. Education in patients with T2DM and follow-up in a training program have useful results and increase the value of the study. The importance of community-based studies is obvious. Internationally recognized training programs and physical activity assessment scales should be validated before being accepted in each society; especially in T2DM. Before implementation of these programs and scales, community-based assessment is required for suitability, both in this study and in others (1-3).

We think that a larger control group should have been included in the study, which was referred to as a limitation. We believe that the power of the study would increase with an appropriately larger control group. Interestingly, there was no comparison with the metabolic status of patients (such as $\mathrm{HbAlc}$ ) and their physical activity status in the study.

We think that patients' cardiac status should have been mentioned in detail in the study. Furthermore, prior guidelines suggested that before recommending a physical activity program, the physician should screen patients with multiple cardiovascular risk factors for coronary artery disease (CAD) in T2DM. However, the screening of asymptomatic diabetic patients for CAD remains unclear, and a recent American Diabetes Association (ADA) consensus statement on this issue concluded that routine screening is not recommended. Certainly, high-risk patients should be encouraged to start with short periods of low-intensity exercise and slowly increase the intensity and duration (4). We think that a study (the present study as well) that investigates the effects of physical activity on diabetic patients should be carried out based on the classification of patients according to their cardiac status and should model physical activity according to this classification, providing better information about the outcome of diabetic patients.
'Beytepe Military Hospital, Department of Internal Medicine, Ankara, Turkey

${ }^{2}$ Gulhane Medical Academy, Department of Cardiology, Ankara, Turkey

${ }^{3}$ Gulhane Medical Academy, Department of Internal Medicine, Ankara, Turkey ${ }^{4}$ Beytepe Military Hospital, Department of Cardiology, Ankara, Turkey

Correspondence to

Mustafa Dinc

Department of Internal Medicine

Beytepe Military Hospital,

Ahlatlibel St. 06800,

Cankaya-Ankara, Turkey

dr.mustafadinc@yahoo.com

Received on Mar/16/2013 Accepted on Apr/29/2013 
Depressive symptoms and comorbidity severity were greater in diabetic patients in the study, and individuals with a sedentary lifestyle had more depressive symptoms. The authors identified that depression treatment could contribute to improve adherence to physical activity. As assessed in a systematic literature review (5), patients with T2DM had a higher incidence of depressive symptoms compared with patients without diabetes; and patients with depressive symptoms had a higher incidence of T2DM. However, the findings supporting the former relationship were weak. As stated in this review, comorbidity is important in patient care, including routine diagnostic approach to find out if another disease is also present (such as depression). As mentioned in another review, the exact nature and direction of this relationship (T2DM vs. depression) is unknown (6). Depression is often thought to be a consequence of diabetes, perhaps caused by the burden of chronic illness. Research has also suggested that depression may be a risk factor for development of diabetes, in part due to biochemical changes caused by depression, and in part because of a reduction of health care behaviors in individuals with depression.

Disclosure: no potential conflict of interest relevant to this article was reported.

\section{REFERENCES}

1. Daniele TM, Bruin VM, Oliveira DS, Pompeu CM, Forti AC. Associations among physical activity, comorbidities, depressive symptoms and health-related quality of life in type 2 diabetes. Arq Bras Endocrinol Metabol. 2013;57(1):44-50.

2. Ferreira SR, Almeida BD, Siqueira AF, Khawali C. [Interventions on the prevention of type 2 diabetes mellitus: is it feasible a population-based program in our country?]. Arq Bras Endocrinol Metabol. 2005;49(4):479-83.

3. BassettDR Jr. International physical activity questionnaire: 12-country reliability and validity. Med Sci Sports Exerc. 2003;35(8):1396.

4. American Diabetes Association. Standards of medical care in diabetes--2013. Diabetes Care. 2013;36 Suppl 1:S11-66.

5. Wagner G, Icks A, Albers B, Abholz HH. [Type 2 diabetes mellitus and depressive symptoms: what is the cause of what? A systematic literature review]. Dtsch Med Wochenschr. 2012;137(11):523-8.

6. Renn BN, Feliciano L, Segal DL. The bidirectional relationship of depression and diabetes: a systematic review. Clin Psychol Rev. 2011;31(8):1239-46. 